\title{
¿Es posible reducir la desigualdad de ingresos en México con la fórmula fiscal de asignación de participaciones actual?
}

\author{
Is it possible to reduce income inequality in Mexico with the current \\ federal resource allocation formula?
}

\author{
Ignacio Ibarra López * \\ Instituto Tecnológico y de Estudios Superiores de Monterrey, México \\ Recibido el 27 de noviembre de 2018; aceptado el 18 de junio de 2019 \\ Disponible en Internet el: 14 de junio de 2019
}

\section{Resumen}

En el presente trabajo se analiza si la forma en que la Federación otorga recursos a los estados en México tiene impacto en la desigualdad de ingresos, medida con el índice de Gini. Se utiliza un modelo de inteligencia artificial, que incluye los siguientes hechos estilizados: a) los criterios de eficiencia y equidad establecidos en la Ley de Coordinación Fiscal (LCF); b) la dinámica de los precios del petróleo y los ingresos federales; c) un modelo de decisión sobre servicios públicos e impuesto estatal, que retoma el planteamiento de Tiebout (1956). El principal resultado del trabajo, es probar que el coeficiente de Gini no disminuye en el tiempo. Por el contrario, tiende a aumentar. Se concluye entonces, que el diseño institucional fiscal que utiliza a la Fórmula Fiscal actual para asignar recursos representa un límite a la reducción de la desigualdad en México.

Código JEL: H21, H23, H77

Palabras Clave: Economía computacional; Federalismo fiscal; Desigualdad; Servicios públicos; Gobiernos subnacionales

\footnotetext{
*Autor para correspondencia.

E-mail address: ignacio_ibarra@msn.com (I. Ibarra López).

La revisión por pares es responsabilidad de la Universidad Nacional Autónoma de México. 


\begin{abstract}
In this paper, we analyze if the way in which the Federation grants resources to states in Mexico has an impact on income inequality, as measured by the Gini index. A model of artificial intelligence is used, which includes the following stylized facts: a) the criteria of efficiency and equity established in the Fiscal Coordination Law (LCF); b) the dynamics of oil prices and federal revenues; c) a model decision on public services and state tax, which takes up the approach of Tiebout (1956). The main result of the work is to prove that the Gini coefficient does not decrease over time. On the contrary, it tends to increase. It is concluded that the fiscal institutional design that uses the current Fiscal Formula to allocate resources represents a limit to the reduction of inequality in Mexico.
\end{abstract}

JEL code : H21, H23, H77

Keywords: Computational economy; Fiscal federalism; Inequality; Public services; Local governments

\title{
Introduction
}

El proceso de descentralización de los servicios públicos iniciado en 1992, replanteó la forma de asignación de los recursos de la federación hacia los estados. El cambio en la Fórmula Fiscal (FF) enunciada en la Ley de Coordinación Fiscal (LCF) buscó incentivar la captación de recursos propios de los estados (criterio de eficiencia), así como, mejorar la equidad distributiva (criterio de equidad). Contrario a lo esperado, casi treinta años después, se observa una tendencia franca hacia una mayor centralización de los recursos. ${ }^{1}$ La dependencia de los recursos asignados por la FF es mayor; en particular hacia las participaciones. Los ingresos propios generados por los estados, representan un porcentaje menor del total de ingresos. ${ }^{2}$ Adicionalmente, las condiciones de desigualdad entre los estados son más profundas.

La literatura que documenta el impacto de la descentralización fiscal en la desigualdad en un país es poco concluyente. Existen trabajos, donde se reconoce que la descentralización fiscal tiene efectos positivos, pues ayuda a disminuir la desigualdad (Tselios et al, 2011; Sepúlveda y Martínez-Vázquez, 2011). Sin embargo, también existe evidencia abundante, de que la descentralización fiscal genera un impacto negativo y puede, de hecho, aumentar la desigualdad (e.g., West y Wong, 1995; Tanzi, 1996; Lessman 2009, 2012).

En el siguiente trabajo se persigue el objetivo de conocer si el arreglo institucional actual (2018), conduce a una reducción de la desigualdad en México, entendida como la reducción

\footnotetext{
${ }^{1}$ Por ejemplo, mientras que en 2006 el porcentaje promedio asignado por la federación a los estados fue de $36.32 \%$, en 2010 se incrementa a 38.18 por ciento.

${ }^{2}$ De acuerdo con los cálculos, en el año 2006, $12.65 \%$ de los ingresos totales de los estados eran generados por su propio esfuerzo recaudatorio mientras que, para 2010, este porcentaje disminuyó a 12.22 por ciento.
} 
del coeficiente Gini en el tiempo. La hipótesis de la que se parte es: "la combinación actual de ponderadores de la Fórmula de Coordinación Fiscal no contribuye a la reducción en el nivel de desigualdad de los mexicanos".

Por arreglo institucional, se entiende a la combinación de parámetros de la fórmula fiscal. Una de las principales aportaciones del trabajo es la de identificar si con un cambio en los parámetros de la citada fórmula, es posible incidir en la desigualdad de ingresos del país en el largo plazo. Otra aportación es la de identificar-si es posible- una combinación óptima de parámetros en la fórmula fiscal. Hasta donde se ha revisado no existe un trabajo en la literatura especializada que haya encontrado dicha combinación.

La metodología que se utiliza es la del denominado enfoque fiscal de incentivos (Oates, 2005, p. 362; Weingast, 2009; Ibarra-Salazar, 2018) donde un sistema fiscal debe ser una institución capaz de materializar las ventajas políticas y las ganancias económicas de la descentralización fiscal, evitando las distorsiones y la desestabilización, que pueden ocasionar restricciones blandas en el gasto y la generación de ingresos.

Para poder operacionalizar este enfoque, se recurre a un modelo computacional que representa el arreglo institucional fiscal de México como un sistema complejo. Dicho modelo recurre al uso de algoritmos de optimización propios de la inteligencia artificial (e.g. escala de colinas, sistemas de clasificación, procesos no ergódicos). De esta forma, se cuenta con un sistema de múltiples agentes que interactúan de forma descentralizada. En este tipo de modelos la interacción genera patrones agregados (i.e. equilibrios o estadios), que no se pueden identificar como una simple suma de conductas de agentes representantivos (Page, 2010). De acuerdo con la revisión de literatura realizada, la incorporación de un modelo como el que se describe al análisis del tema propuesto, implica la tercera contribución a la literatura que se realiza con el trabajo.

El artículo se divide en cinco apartados. En el primero se describen los elementos teóricos del modelo de descentralización fiscal y las características del modelo. Se analiza la literatura sobre la desigualdad, el crecimiento y otras variables, así como la pugna entre gobiernos subnacionales (i.e. estados y municipios) y la federación. En el segundo apartado, se discuten los hechos estilizados del proceso fiscal en México. En particular se describen los módulos de entorno del modelo: la dinámica de los precios del petróleo y la fórmula de coordinación fiscal. En el tercer apartado, se presenta el modelo y su utilidad para probar hipótesis sobre el impacto de la FF sobre la desigualdad del ingreso. La cuarta sección presenta los resultados, las inferencias y la dinámica generada por el modelo. La última sección concluye. 


\section{Antecedentes y objetivos}

\section{Las funciones fiscales del gobierno y la desigualdad}

El proceso de descentralización federal puede describirse a partir de las funciones fiscales del gobierno: 1) la provisión óptima de bienes públicos; 2) la estabilización macroeconómica; 3) la distribución del ingreso-véase Musgrave y Musgrave (1989). Desde su concepción, existe un debate en torno a la conveniencia de descentralizar, sin que exista hasta el momento evidencia concluyente. Por un lado, se asume que transferir autoridad a los gobiernos subnacionales, mejora la provisión de bienes públicos y esto, a su vez, mejora la eficiencia del gobierno en su conjunto (Bardhan, 2002). Los gobiernos subnacionales, tienen un mayor conocimiento de las necesidades de la población y, por tanto, son capaces de determinar de mejor forma las combinaciones de impuestos y servicios públicos. Sin embargo, también se ha reconocido que en la asignación de bienes públicos en una sociedad, es imprescindible la participación de un gobierno central (Oates, 1968).

Por su parte, respecto a la función de estabilización, destacan los estudios que la vinculan con el crecimiento económico. Un argumento recurrente, es que la descentralización incrementa la competitividad entre las regiones y produce asignaciones óptimas, sin recurrir a la intermediación de un gobierno centralizado (ver Careaga y Weingast, 2003).

Rodríguez-Pose y Krøijer (2009), muestran que el traslado de los impuestos a gobiernos subnacionales, puede tener efectos positivos en el largo plazo. En opinión de los autores, un gobierno subnacional que controla su fuente de recursos, responde mejor a las demandas de la población, lo que incrementa el desempeño económico en su territorio. Evidencia a favor de este argumento se ha encontrado al menos para los países de la OCDE en Rodríguez-Pose y Ezcurra (2010).

La literatura sobre la función fiscal de distribución de ingresos es abundante, no concluyente y con una mayor evidencia en relación a que la descentralización aumenta la desigualdad. $\mathrm{El}$ argumento de mayor peso a favor de la descentralización, es que reduce la desigualdad del ingreso (Tselios et al., 2011). Estudios sobre la dinámica de la relación descentralización-desigualdad en países de Latinoamérica (ver a Sepúlveda y Martínez-Vázquez, 2011), encuentran efectos mixtos. por un lado, se incrementa la pobreza, pero al mismo tiempo (y bajo ciertas condiciones), es posible disminuir la desigualdad de ingresos (e.g. solo si el gobierno representa el 20\% del valor del PIB o más).

Se ha encontrado también que los procesos de descentralización tienen efectos negativos en la distribución del ingreso, generando mayor desigualdad (e.g., Tanzi, 1996). El impacto negativo de la descentralización sobre la desigualdad ha sido reportado entre otros por Zhang (2006), quien además muestra que en el proceso de descentralización en China, los costos de 
transacción asociados a la descentralización han contribuido a generar un gobierno excesivo, lo cual se ha traducido en impuestos más altos.

Paralelamente, se observa que quienes pagan más son los contribuyentes de zonas rurales y pobres. En contraposición, los contribuyentes de zonas ricas disfrutan de subsidios y menores impuestos. Así, la descentralización fiscal promueve impuestos con lógica regresiva y explican la divergencia regional en materia de crecimiento económico. La conclusión de este estudio es que en China, la política de descentralización fiscal ha conducido a mejorar las condiciones de las unidades territoriales más ricas, incrementando la brecha de desigualdad con las más pobres.

Prud'homme (1995) argumenta, a su vez, que un gobierno centralizado tiene una mayor capacidad para realizar compensaciones entre unidades territoriales en comparación con un gobierno descentralizado. La descentralización conlleva a un proceso de competencia fiscal entre unidades territoriales, donde las regiones más ricas atraen más factores móviles de la producción (debido a que tienen mejor infraestructura física, capital humano, acceso mercados más desarrollados y por lo tanto costos más bajos). La consecuencia de incentivar la competencia a través de la descentralización, es que las unidades territoriales ricas tenderán a ser más ricas y las regiones pobres tenderán a ser más pobres, provocando una mayor desigualdad.

En síntesis, la literatura reconoce (sin ser concluyente) que la descentralización puede ayudar a estabilizar fiscalmente a un país, mejorar la dotación de servicios de públicos y bajo ciertas condiciones particulares, disminuir la desigualdad de ingresos (incluso en países de Latinoamérica). Sin embargo, también ha encontrado que un mismo proceso de descentralización tiende a generar una mayor desigualdad de ingresos. A continuación se describe el proceso mediante el cual se transfieren recursos fiscales en México a los gobiernos subnacionales (en particular a las entidades federativas).

\section{Sistema de transferencias intergubernamentales en México}

Según Blöchliger (2014), un sistema de transferencias intergubernamentales debe ser un instrumento diseñado para canalizar recursos financieros a los gobiernos subnacionales (i.e. estados y municipios). La finalidad es ofrecer a sus ciudadanos niveles similares de servicios públicos con niveles similares de esfuerzo de tributación. En ese sentido, para el caso de México, se ha creado la Ley de Coordinación Fiscal (LCF) con sus últimas modificaciones en $2018^{3}$. Dicha ley establece dos instrumentos: las participaciones y las aportaciones. Las primeras, son calculadas sobre el Fondo General de Participaciones (FGP), en el cual los criterios de asignación son de tipo poblacional y de eficiencia recaudatoria. Por su parte, las

${ }^{3}$ Véase: http://www.diputados.gob.mx/LeyesBiblio/pdf/31_300118.pdf 
aportaciones federales se asocian con los recursos que dependen de proyectos específicos que los estados deseen realizar. Esto implica que en cada fondo se incluyen criterios específicos tanto para obtener recursos como para comprobar su ejercicio.

Para saber cuánto se asigna a cada estado, se emplea una fórmula la cual se ha analizado en diferentes trabajos los cuales enuncian problemas tales como la generación de una mayor dependencia del gobierno federal, la complejidad en el cálculo, el que se puede favorecer la opacidad en el otorgamiento de recursos y el posible incremento de la desigualdad (Salazar et al, 1999; Chavez-Presa, 2004; Sobarzo, 2004; OCDE, 2007; Hernández-Trillo, 2011; Figueroa, 2014; Rodríguez-Pueblita, 2017). En particular, se han identificado como fuentes de la desigualdad de ingresos, los recursos transferidos por convenios específicos entre la federación y las entidades federativas, así como las participaciones que dependen en buena parte de la actividad petrolera.

Sin embargo, también se ha encontrado como una ventaja, el que existan transferencias condicionadas de la federación hacia los estados. Por ejemplo, en Carmona y Caamal-Olvera (2018) se presenta evidencia de que la descentralización reduce la desigualdad salarial en particular cuando se toma en cuenta la asignación a través de recursos etiquetados.

Díaz-Cayeros (2004), proporciona una de las explicaciones más concretas sobre el origen y consecuencias de mantener la fórmula fiscal, así como el proceso de otorgamiento de recursos de la federación hacia los estados en México. Según este autor en un sistema democrático, las luchas presupuestarias son la expresión de los acuerdos políticos que buscan mantener el equilibrio entre pagar impuestos, generar servicios públicos y la relación que tienen los políticos con sus gobernados.

En el arreglo federal mexicano, los gobiernos estatales no necesitan gastar de acuerdo con un equilibrio de ingresos recaudados y bienes y servicios provistos. De forma alternativa, elaboran presupuestos en función de lo que pueden obtener de las transferencias federales en el presente (vía participaciones o transferencias) o en el futuro (a través de deuda).

Para presionar a la federación, los estados utilizan a los diputados federales electos que conforman el Congreso de la Unión, el cual tiene como una de sus principales atribuciones el modificar y en su caso autorizar el presupuesto que plantea el Ejecutivo Federal. En la misma línea Rodríguez (2006) destaca que la disputa por el presupuesto federal se plasma en formas de negociación formales, tales como la Asociación Nacional de Gobernadores (ANAGO), posteriormente identificada como Conferencia Nacional de Gobernadores (CONAGO).

\section{Servicios públicos e impuestos}

Conocer la relación endógena entre la generación de servicios públicos y los impuestos que cobra el gobierno, es fundamental para entender cómo un arreglo institucional de transferen- 
cia de recursos, modifica la desigualdad de ingresos. Al respecto, Tiebout (1956) concebía que la dotación de servicios públicos era el resultado de un modelo en el que, a partir de una competencia por contribuyentes entre unidades territoriales.

El ordenamiento de Tiebout funciona de la siguiente manera: cada unidad territorial ofrece un conjunto de bienes y servicios públicos (nivel de servicio) a diferentes precios (impuestos), lo cual genera una oferta de bienes y servicios públicos diversa (i.e., las combinaciones de cada unidad territorial entre impuesto y nivel de servicio público son diferentes).

Se asume que los impuestos y el nivel de servicios públicos guardan una relación positiva. La información del nivel de servicios públicos y de los impuestos, le permite a los contribuyentes realizar valuaciones del costo y el beneficio que obtendrían por establecerse en una unidad territorial en particular. Con estas valuaciones, los contribuyentes tenderán a moverse de un lugar a otro, hasta que encuentren la combinación óptima en relación al nivel de servicio público e impuesto que pagan. Así, se motiva un proceso de autoselección de los individuos, que revelaría sus preferencias de servicio público e impuesto, generando agrupamientos de contribuyentes con preferencias similares. Esta es la razón por la que el modelo también sea conocido como "votar con los piés" (foot-voting).

El resultado final que anticipa Tiebout, es Pareto superior a cualquier otra asignación que se realice, porque le permite a cada contribuyente establecerse en el lugar que más se ajusta a sus preferencias, revelando así la información sobre la tasa de impuestos y el nivel de servicios públicos.

En la literatura existe una buena cantidad de artículos donde se citan o analizan las principales hipótesis de Tiebout, ${ }^{4}$ los cuales hasta el momento enfrentan una fuerte controversia en torno a su validez (Hoyt, 1991; Orfield, 2002). Parte de las críticas puede originarse por el reto que implica replicar la lógica de este modelo con herramientas matemáticas y estadísticas convencionales (i.e., econometría, ecuaciones diferenciales o teoría de juegos). ${ }^{5}$

De forma alternativa, se han propuesto modelos Tiebout computacionales como el de Kollman, Miller \& Page (1997). En este modelo, se pueden realizar comparaciones directas entre diferentes tipos de instituciones políticas (e.g., referendum y representación proporcional), para analizar la relación entre inestabilidad política y la heterogeneidad de las preferencias de votantes.

En Penn (2004), se utiliza un modelo Tiebout computacional para explicar la fragmentación en ciudades. Se modela la heterogeneidad en los ingresos, instituciones políticas y la posibilidad de que cada ciudad decida discrecionalmente la tasa de impuestos.

El modelo busca explicar cómo es que se definen nuevas unidades territoriales considerando que la secesión de territorios es posible (i.e. una ciudad puede ceder territorio a otra

\footnotetext{
${ }^{4}$ Por ejemplo, Dowding, John y Biggs (1994) presentan un análisis de los hallazgos de 200 trabajos asociados al modelo Tiebout. ${ }^{5}$ Por ejemplo en Sakashita (1999) se plantea un sistema de ecuaciones dinámicas con solución mediante métodos numéricos.
} 
ya existente o de nueva creación). Una conclusión importante, es que al incrementar la tasa de impuestos, se incrementa también el número de unidades territoriales, la heterogeneidad en el ingreso promedio de sus habitantes y el bienestar social (i.e. nivel de servicios). En otras palabras, el modelo de Penn prueba computacionalmente el supuesto de Tiebout: mayor competencia entre unidades territoriales, mejora el nivel de servicios públicos.

En Nishida et al, (2011) se utiliza el modelo Toebout para analizar fenómenos migratorios. Su modelo incluye dos tipos de agentes: 1) gobiernos subnacionales (e.g estados de una federación, departamentos), los cuales determinan políticas presupuestales en torno a los servicios que deben brindar y; 2) ciudadanos, que deciden migrar en función del nivel de servicios y de impuestos. Los gobiernos subnacionales tienen tres estrategias: 1) mantener un servicio constante; 2) imitar la mejor estrategia del gobierno que atrae la mayor cantidad de ciudadanos y; 3 ) generar un nivel de servicios en base a las preferencias de los ciudadanos. El trabajo tiene como conclusión principal que cuando cada gobierno subnacional tiene su propia estrategia, se favorecen los procesos migratorios. Es decir, la competencia entre unidades territoriales genera migraciones. Sin embargo, la convergencia de estrategias entre gobiernos subnacionales (i.e. que todos cobren lo mismo o generen el mismo nivel de servicios públicos), disminuye la migración. Por lo anterior, Nishida et al, (2011) prueba también la validez del argumento de Tiebout en torno a que una mayor competencia genera una oferta más diversa de servicios públicos.

En el presente trabajo, al modelo Tiebout se le agrega el arreglo institucional de transferencia de recursos de la federación comentado en el apartado 1.2, así como algunos hechos estilizados del proceso de generación de ingresos fiscales que a continuación se discuten.

\section{Métodos, materiales empleados y fuentes de información}

\section{Hechos estilizados del sistema de transferencias intergubernamentales en México}

La repartición de recursos a través de la Fórmula Fiscal (FF)

Según el artículo 2 de la LCF, el Fondo General de Participaciones (FGP) se reparte entre las diferentes entidades del país (32), asignándole a cada una la fracción $P_{i, t}$ calculada bajo la regla siguiente:

$$
P_{i, t}=P_{i, 07}+\Delta F G P_{07, t}\left(0.6 C 1_{i, t}+0.3 C 2_{i, t}+0.1 C 3_{i, t}\right.
$$


Donde $\mathrm{P}_{i, 07}$ son las participaciones del estado $i$ en el año de 2007, $\Delta F G P_{07, t}$ es el cambio en los recursos del FGP de 2007 al año $t, C l_{i, t}$ es un coeficiente que asigna recursos a la entidad $i$ en el período $t$, el cual depende de relaciones de los cambios del Producto Interno Bruto (PIB), así como del tamaño de la población. Los coeficientes $C 2_{i, t}$ y $C 3_{i, t}$, se calculan con la información relacionada con la recaudación de impuestos y derechos que percibe la entidad $i$ en el año $t$ (tomando como fuente los datos de la última cuenta pública oficial). El coeficiente $C 1_{i, t}$ representa al criterio de desigualdad, mientras que $C 2_{i, t}$ y $C 3_{i, t}$ son criterios de eficiencia recaudatoria ${ }^{6}$.

El efecto poblacional se incluye en todos los coeficientes de la fórmula $\left(C_{i, r^{\circ}} C 2_{i, t} y\right.$ $C 3_{i, t}$ ), dado que el PIB como los impuestos que se generan para entidad $i$, están relacionados positivamente con el crecimiento poblacional. Por esta razón, no es de extrañar que estados con mayor actividad económica (PIB e impuestos) sean aquellos que terminan concentrando la mayor cantidad de recursos participables en México.

Adicionalmente, se debe considerar el "efecto político" identificado en el trabajo de Díaz-Cayeros (2004) en el cual también a su vez existe un efecto poblacional, pues la cantidad de diputados federales también depende de la cantidad de habitantes en un estado del país. La FF plantea ponderaciones específicas, i.e., 0.6 para $C 1_{i, t}, 0.3$ para $C 2_{i, t}$ y 0.1 para $C 3_{i, t}$ que muestran la importancia que tiene cada criterio en la asignación de recursos.

La otra fuente de ingresos para los estados son las aportaciones federales, las cuales dependen de criterios específicos tanto para la asignación como para la comprobación. Incluir todas y cada una de las condiciones que plantea la LCF para cada fondo de aportaciones, sería un proceso mucho más complicado de modelar, por lo cual en el modelo computacional se omite la asignación de recursos a los estados por esta vía. Se reconoce esta omisión como una limitante del trabajo y como una mejora a futuro de dicho modelo.

\section{Ingresos públicos y los precios del petróleo}

El precio del petróleo es una variable clave en las finanzas públicas del país que determina los recursos participables a los estados, $\mathrm{Al}$ analizar el período de enero de 1990 a abril de 2011 (antes de la implementación de reformas de liberalización del sector energético), la contribución de los ingresos petroleros en los ingresos del gobierno mexicano a lo largo del tiempo, observamos que, durante el período de análisis, los ingresos petroleros han representado entre $25 \%$ y $33 \%$ del total de ingresos públicos del país (figura 1).

\footnotetext{
${ }^{6}$ Para un mayor detalle de los componentes C1, C2 y C3 de la Fórmula Fiscal se puede consultar la última versión de la LCF (30/01/2018).

Disponible en: http://www.diputados.gob.mx/LeyesBiblio/pdf/31_300118.pdf
} 


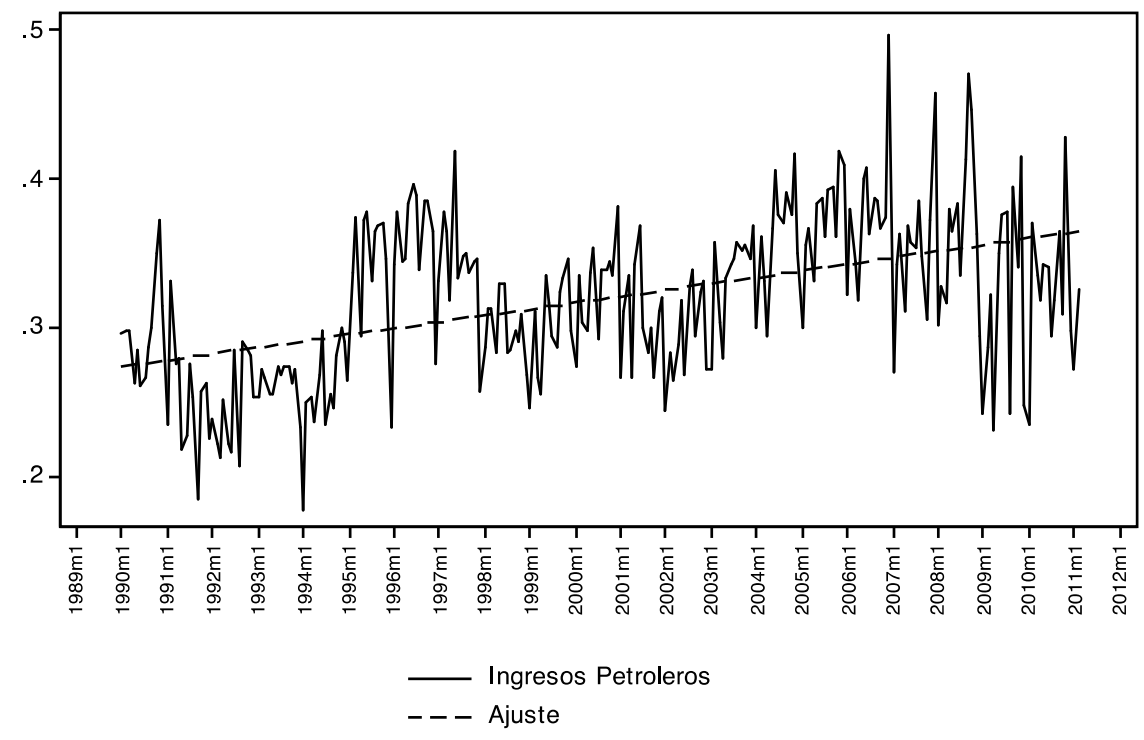

Figura 1.Contribución de los ingresos por petróleo, enero 1990-abril 2011.

Fuente: elaboración propia con datos de Estadísticas Oportunas de Finanzas Públicas y Deuda Pública. Ingresos Presupuestarios Del Sector Público (Dirección General Adjunta de Estadística de la Hacienda Pública,

Unidad de Planeación Económica de la Hacienda Pública, SHCP). Disponible en: http://www.shcp.gob.mx/ POLITICAFINANCIERA/FINANZASPUBLICAS/Estadisticas_Oportunas_Finanzas_Publicas/Paginas/unica2.aspx

Después de la llegada del presidente Enrique Peña Nieto ${ }^{7}$, la cantidad de recursos petroleros que se consideran para calcular las participaciones incluso aumentó hasta ocupar un $78.47 \%$ en 2018.

Con el fin de investigar la sensibilidad de los ingresos federales a las variaciones del precio del petróleo y capturar esta característica de manera formal, se propone un modelo de series de tiempo capaz de explicar el crecimiento de los ingresos federales en función de dos variables: 1) el precio spot del petróleo y; 2) el Impuesto Especial sobre Productos y Servicios (IEPS).

El modelo que mejor ajusta y que arroja residuos independientes e idénticamente distribuidos, es un proceso ARMA $(12,1)-\mathrm{ARCH}(1) .{ }^{8}$ Los resultados muestran que un crecimiento

\footnotetext{
${ }^{7}$ Según la Ley de Coordinación Fiscal (LCF) antes de la modificación de 2014, los recursos participables comprenden todos los impuestos federales, así como por los derechos sobre la extracción de petróleo y de minería (LCF, Art. 2, reforma al 24 de junio de 2009). A partir de 2014 se han incorporado las reformas sobre ingresos de hidrocarburos que marcan porcentajes específicos sobre dichos ingresos (e.g. en 2015 se plantea el 73.00\%, para 2016 el $74.82 \%$, en $201776.65 \%$ y en 2018 el 78.47 por ciento. En relación a la Fórmula Fiscal no se plantearon cambios en los parámetros.

${ }^{8}$ Se estimaron varios modelos ARMA(p,q) con volatilidad condicional autoregresiva tipo GARCH (ver Bollerslev, 1990). El orden final del modelo se elige en base a los Criterios de Información de Akaike y Bayes. Resultados detallados de estas estimaciones y
} 
$1 \%$ del precio spot del petróleo, genera un incremento estadísticamente significativo de $16.5 \%$ de los ingresos federales en promedio. Este hallazgo es congruente con análisis anteriores como el de Eifert et al (2003).

\section{Estructura del modelo computacional}

El modelo computacional que se utiliza en este trabajo, integra módulos que caracterizan dos tipos de agentes: estados y contribuyentes. Cada uno de estos, tiene características específicas y obedece a ciertas reglas de decisión. En adición, se incluyen módulos de entorno en los que se introduce la dinámica que siguen los precios del petróleo y la asignación de participaciones mediante la fórmula fiscal. A continuación se describen cada uno de estos módulos. ${ }^{9}$

\section{- Agentes \\ Estados}

Las entidades federativas de México en el modelo, se representan como unidades territoriales, que pueden ser habitadas por los agentes, $\mathrm{y}$ que son gobernadas por una autoridad que decide el nivel de servicios públicos y la tasa impositiva. Se utiliza un Autómata Celular (AC), el cual se puede entender como un sistema espacial dinámico con los siguientes componentes (Von Neumann y Burks, 1966): 1) una rejilla bidimensional, en la cual cada celda representa algún tipo de agente; 2) un conjunto de posibles condiciones en la que se encuentra cada elemento de la rejilla; 3) reglas de evolución, que permiten la transición de una condición a otra, y que dependen de la interacción con otros agentes así como de condiciones que se tenían anteriormente; 4) una vecindad definida para cada agente; 5) un 'reloj' que indica a cada agente, cuando deben aplicarse las reglas de evolución.

Cada elemento de la rejilla del AC, representa una parte del total del territorio de la entidad federativa. Se tienen un total de 3,200 celdas que conforman los 32 estados de México (i.e. cada entidad federativa tiene 100 unidades de territorio). Los individuos en cada entidad federativa, enfrentan la misma tasa impositiva y nivel de servicio público que defina la autoridad. Se parte del supuesto, de que las decisiones tomadas desde un gobierno estatal, son acatadas plenamente en todo el territorio. Adicionalmente, en cada entidad federativa se

criterios de decisión están disponibles con los autores.

${ }^{9}$ El lector puede tener acceso al programa contactando directamente al autor. Note que es importante tener instalado Java v. 7 o mayor. Es preferente utilizar Mac OSX 10.7.5 para usuarios de Safari o Windows 7 para usuarios de Goolge Chrome o Internet Explorer en su versión más reciente. El usuario puede examinar escenarios diversos como el impacto de cambio en distintas variables sobre la desigualdad del ingreso, así como cambiar condiciones iniciales y observar la dinámica poblacional de los contribuyentes en los estados de la República Mexicana.. 
calcula el costo total del servicio público, así como los impuestos estatales recaudados y el PIB estatal. Estas dos últimas variables sirven para determinar las participaciones asignadas a cada estado $i$ en los $t$ períodos en los que se desarrolla la simulación $\left(P_{i, t}\right)$.

Finalmente, para cada estado se determina el presupuesto, el cual incluye los ingresos (recaudación estatal y participaciones) y egresos (costo de la oferta de servicios públicos). El costo de oferta de bienes públicos, se obtiene multiplicando el nivel de servicio público establecido en el estado, por la cantidad de agentes que se encuentran en su territorio.

\section{Contribuyentes}

Cada estado, es habitado por un número de contribuyentes, el cual es determinado incialmente por un parámetro exógeno de población $(P o b)$. Cada agente, puede ocupar solo una rejilla al mismo tiempo. El AC, puede estar densamente poblado (población cercana al 100\%) ,o escasamente poblado (población cercana al 0\%).

A su vez, todos y cada uno de los $j$ contribuyentes, poseen características específicas. Esta condición genera una población heterogénea: 1) un nivel de ingreso; 2) el nivel de disfrute (preferencia) del servicio público y, 3 ) la utilidad que derivan los agentes $\left(U_{j, t}\right)$. Esta última de carácter endógeno, ya que se encuentra en función del nivel de servicio público, el ingreso y la tasa de impuestos.

\section{- Módulos de decisión}

\section{Estados}

En cada período de la simulación, los estados definen el nivel de provisión del servicio público $\left(S_{i, t}\right)$, en base a las preferencias de los ciudadanos. A su vez, las preferencias y la demanda individual de cada ciudadano se obtiene mediante una función de utilidad Cobb-Douglas, tal cual lo propone Penn (2004). La tasa impositiva $\left(T_{i, t}\right)$, depende del nivel de ingreso del individuo $j$. Una vez que cada estado ha establecido el nivel de provisión del servicio público, éste puede ser disfrutado por todos los ciudadanos que se encuentran en su superficie, siempre que haya disponibilidad de espacio (i.e. no se ha ocupado completamente el territorio) en la entidad federativa.

Con los datos de provisión de servicios y tasa impositiva, se calcula la recaudación del estado $i$ en el período $t$. En cada estado se calcula también un balance en las finanzas públicas $\left(B_{i, t}\right)$. Dicha variable, está en función de lo que se recauda de los contribuyentes, de las participaciones que reciben los estados de la Federación y del costo de proveer el nivel de servicios $S_{i, t}$ Los estados modifican el nivel de provisión del servicio y la tasa impositiva, de 
acuerdo con los cambios que experimenta este balance financiero. Si el valor de $B_{i, t}$ permite proveer el servicio, los estados adoptan los valores del servicio $S_{i, t}$ y la tasa impositiva $T_{i, t} \cdot \mathrm{Si}$ esto no es posible, mantienen los niveles del periodo anterior, i.e., $S_{i, t-1}$ y $T_{i, t-1}$.

\section{- Cambios de residencia}

En cada período, los agentes son capaces de cambiar de residencia. Este cambio, es el resultado de un análisis costo beneficio entre el lugar de residencia actual, y otro estado con mejores tasas impositivas. El algoritmo que genera estos cambios es el siguiente: al inicio de cada período, se calcula el ingreso $W_{j, t}$ del contribuyente $j$ en el momento $t$, el cual se asocia positivamente con el nivel de servicio público y negativamente con el pago de impuestos estatales y federales.

En cada período, se determina el ingreso que obtiene el individuo en el estado donde reside, y se escoje aleatoriamente una celda en otro estado donde la tasa impositiva es más baja. Si el ingreso potencial en el estado de comparación es mayor al ingreso de residencia, se genera un movimiento del agente a la celda seleccionada, siempre que la celda no se encuentre ocupada por otro agente (para reflejar límites físicos a la provisión del servicio).

El cambio de residencia depende de una probabilidad calculada a partir del Censo de Población y Vivienda 2010 del Instituto Nacional de Estadística Geografía e Información (INEGI). Con ello, se busca incluir aquellos factores idiosincráticos asociados a los cambios de residencia en México (e.g., se observa que ciertos estados son elegidos en mayor medida), así como los costos de traslado y transacción, presentes en un cambio de residencia. ${ }^{10}$

\section{- Módulos de entorno}

En adición a los módulos de decisión, existen módulos que reflejan las condiciones específicas del proceso de generación de recursos públicos en México. En concreto, el movimiento en precios del petróleo y la asignación de participaciones utilizando la FF descrita en LCF. Se incluyen todos los ingresos participables que indica la LCF, utilizando los datos agregados del Fondo General de Participaciones (FGP). Lo que no se ha incluído en todo caso, son las aportaciones federales, las cuales se asocian a recursos para realizar programas y proyectos específicos.

\footnotetext{
${ }^{10}$ Se ha utilizado información de INEGI para la estimación de estas probabilidades http://www.inegi.org.mx/est/contenidos/proyectos/ccpv/cpv2010/Default.aspx. Se puede consultar directamente con el autor para mayor información.
} 


\section{Precios del petróleo}

A fin de reflejar el impacto de los movimientos del precio del petróleo sobre los ingresos federales, se utiliza el modelo de reversión a la media descrito en Schwartz (1997), y se utiliza la fórmula de asignación de participaciones que se describe en la sección 2.1 de este documento.

Fórmula de asignación de participaciones de los estados

El proceso de cálculo de la fórmula fiscal que se ha integrado al modelo tiene algunas particularidades:

1) $P_{i, 07}$ equivale a $P_{i, t-1}$ en el modelo, i.e., el monto de participaciones generadas en las simulaciones en el período $t-1$ para el estado $i$.

2) El Fondo General de Participaciones (FGP), representa 20\% del total de los recursos participables en cada periodo. Los recursos participables que se contemplan en el modelo, serán el total de ingresos por el impuesto sobre la renta que pagan los contribuyentes y los ingresos petroleros.

3) $\Delta F G P_{07, t}$ equivale a $\Delta F G P_{t}=F G P_{t}-F G P_{t}-1$ en el modelo, donde $F G P_{t}$ es el FGP en el período $t$ y $F G P_{t-1}$ en el período anterior.

4) La fórmula fiscal requiere el cálculo del PIB a nivel estado $i$, en diferentes períodos de tiempo $t\left(P I B_{i, t}\right)$. La suma de los ingresos de todos los habitantes del estado $i$, resulta en la estimación del PIB.

5) El nivel de recaudación para cada entidad en los diferentes periodos, será el ingreso obtenido de multiplicar la tasa impositiva por el ingreso del contribuyente, i.e., $T_{i, t} W_{j, t}$

6) El INEGI presenta información sobre la población en los estados cada 5 años (conteo) y 10 años (censo). En el modelo, la población se conoce en cada periodo.

7) Los parámetros y valores iniciales que acompañan a C1i,t, C2i,t, C3i,t son pond $1=0.6$, pond $2=0.3$ y pond $3=0.1$. Como se verá en la siguiente sección, estas son las variables de elección del modelo y el coeficiente de Gini será la función objetivo. La variación de estos parámetros permite generar escenarios contrafactuales para evaluar la conveniencia de diferentes esquemas de asignación de recursos y reducción de la desigualdad. 


\section{Desarrollo del modelo}

El modelo está diseñado para generar resultados para cada periodo $t$, para un horizonte temporal $T$ y tiene las siguientes características:

1) En el périodo $t=0$ de cada simulación, se definen los parámetros iniciales de entorno (e.g., la población, los ponderadores de la Fórmula Fiscal, entre otros). Se determina también las características que tienen los agentes en cada entidad federativa (i.e. parámetros de su función de utilidad).

2) Para el caso del ingreso, se utiliza la información de la ENIGH 2010. Debido a la cantidad de agentes que integran la simulación, se podrían generar valores muy altos de ingresos. A fin de evitar este problema, se ha construido un ingreso normalizado para cada decil, donde el valor que puede tomar un individuo en el decil más bajo es 0.1602 , y el valor si pertenece al decil más alto es $2^{11}$.

3) En cuanto a los procesos de migración, se utiliza información del Censo 2010 de INEGI de dos grupos de variables: la condición de migración y el lugar de residencia en el año 2000. En los censos que realiza el INEGI, existe también información de la migración de las personas en tres categorías: migrante estatal, migrante internacional y no especificado. Con estos datos, se calcula una probabilidad de que las personas en una entidad federativa, decidan cambiar de residencia utilizando una matriz de Markov.

4) La migración interestatal solo considera los tres principales destinos para cada contribuyente (i.e. los estados con la probabilidad de atracción más alta).

5) Se definen las características iniciales para las 32 entidades federativas en México, en relación a presupuestos iniciales, nivel de servicio público e impuesto que definen las autoridades.

6) En cada período se actualiza el precio del petróleo, asumiendo un proceso dinámico como el que documenta Schwartz (1997). Se utiliza un precio inicial en cada simulación, que representa el promedio de los precios del período que analizamos en la sección 2.2 (i.e. de enero de 1990 a abril de 2011).

7) Posteriormente, se determina para todas y cada una de las entidades federativas, el presupuesto con que cuentan periodo a periodo, integrando por un lado los recursos

\footnotetext{
${ }^{11}$ Por cuestiones de espacio no fue posible incluir la tabla de deciles. Para conocer de forma detallada este cálculo se recomienda contactar directamente al autor.
} 
federales $3 / 4$ asignados por la $\mathrm{FF}^{3} / 4 \mathrm{y}$, por el otro, los recursos de origen estatal se logran recaudar de los contribuyentes.

8) En base a su presupuesto, cada entidad federativa determina el nivel de servicio público y de impuesto, utilizando las reglas de decisión antes descritas.

9) Los agentes, toman la decisión de quedarse o no en la entidad federativa, dado el nivel de servicio público, los impuestos determinado por los estados, y utilizando su función de utilidad. Si se gana una mayor utilidad con el cambio, el agente se cambia de residencia.

10) Finalmente, se generan las gráficas y valores en las diferentes variables de desempeño.

La Figura 2 muestra dos imágenes del modelo que incorporan las 32 entidades federativas. Estados con mayores impuestos, tienden al color negro y estados con menores impuestos, tienden al color blanco. Cada estado esta “poblado" por agentes (puntos en color blanco o negro).

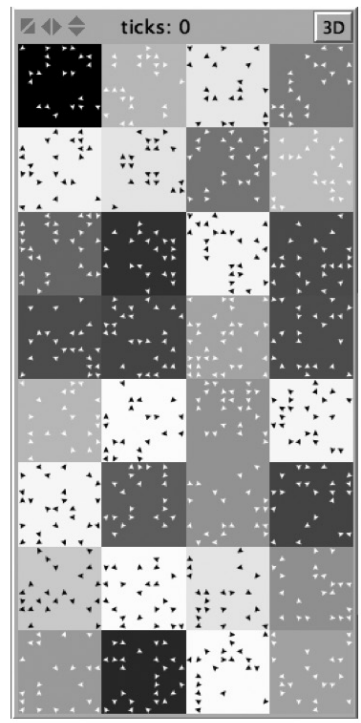

(a) AC' al inicio

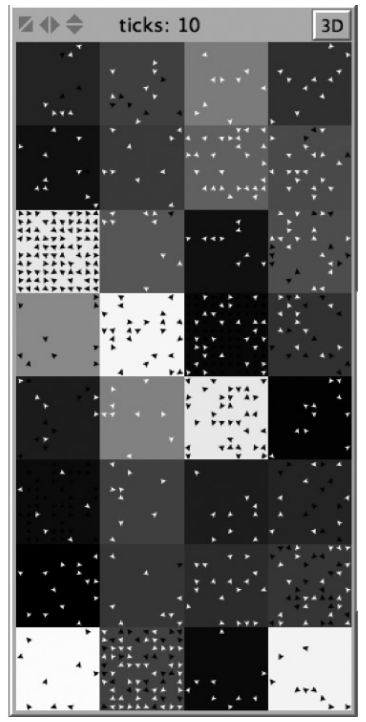

(b) AC con adaptacion

Figura 2. Representación gráfica del modelo. 
La Figura 2 (a) muestra a los estados en el momento 0 (condiciones iniciales del modelo). En la figura 2 (b), se muestra el modelo una vez que se ha generado un proceso de adaptación en el tiempo y reflejando la distribución poblacional de México. Este segundo caso se genera por las decisiones de residencia de los agentes.

En la figura 2(b), se observa que se ha producido un cambio en el color de varios estados en función del ajuste de su política impositiva. También se observan cambios en la concentración poblacional de ciertos estados, en función del nivel de servicio público y de impuesto. En este caso, el nivel de servicio público es un valor adimensional, que representa la demanda de cualquier servicio público (e.g. número de policías, total de lámparas, calles pavimentadas). La idea, es introducir una variable que nos permita reproducir la lógica del modelo Tiebout, sin que esto implique una mayor sofisticación.

\section{Hipótesis de trabajo}

La hipótesis planteada es que: "la combinación actual de ponderadores de la Fórmula de Coordinación Fiscal no contribuye a la reducción en el nivel de desigualdad de los mexicanos". Para probar dicha hipótesis y contrastar resultados, se incorporan las siguientes variables de desempeño al modelo: 1) coeficiente de Gini en función del ingreso de los ciudadanos, el cual es una variable proxy del bienestar social; 2) porcentaje de ciudadanos que deciden cambiar de residencia en relación al total de ciudadanos y; 3) el impuesto estatal promedio en todos los estados.

El valor de cada una de estas variables de desempeño, es un resultado que depende del conjunto de parámetros iniciales (i.e. las ponderaciones de la fórmula fiscal). De esta forma, el análisis, se concentra en conocer el efecto que tienen diferentes combinaciones de pond1, pond 2 y pond 3 sobre estas variables, y en particular sobre la el índice de Gini.

Para probar la hipótesis, planteamos el experimento siguiente que consta de:

1. Escenario actual: se realizan $100^{12}$ simulaciones de 144 periodos mensuales (12 años) con pond $1=0.6$, pond $2=0.3$ y pond $3=0.1$ definidos en $t=0$. Cada simulación empieza con las mismas condiciones iniciales (ver tabla 1).

2. Escenario optimizado: el objetivo es encontrar el valor del coeficiente de Gini que corresponde a una combinación de ponderadores de la FF óptimos (pond 1 *, pond $2 *$ y pond $\left.3^{*}\right)$. Utilizando el algoritmo de optimización escalada de colina (Hill-Climbing),

\footnotetext{
${ }^{12} \mathrm{El}$ modelo es capaz de generar cualquier número de simulaciones que se requiera. $\mathrm{Al}$ aumentar el número de repeticiones (e.g., a 1,000 repeticiones) no se observaron en realidad cambios significativos en los resultados.
} 
se corren 100 simulaciones, y se escoje aquella combinación que minimiza el coeficiente de Gini de largo plazo (al final del periodo 144). Cada simulación, parte de los mismos valores iniciales de la tabla 1, pero de diferentes combinaciones en pond1, pond2 y pond3. Los ponderadores son las variables de elección.

3. Una vez ubicada la combinación óptima de ponderadores, se generan otras 100 simulaciones, manteniendo constantes los valores de pond $1^{*}$, pond $2 *$ y pond $3 *$. Con estos resultados, se calcula el valor promedio óptimo que puede tener el coeficiente de Gini.

4. Finalmente se comparan los resultados de ambos modelos; es decir, el de la combinación óptima (pond $1 *$, pond $2 *$ y pond $3 *$ ), y el del arreglo institucional que actualmente existe en la FF (pond $1=0.6$, pond $2=0.3$, pond $1=0.1$ ).

Tabla 1

Combinación de parámetros en condiciones iniciales $(t=0)$

\begin{tabular}{cc}
\hline Parámetro & Valor en $\mathrm{t}=0$ \\
\hline Precio inicial del petróleo & $\$ 25.560$ \\
Tasa de impuestos federales & $30.000 \%$ \\
\hline
\end{tabular}

Fuente: Elaboración propia con datos de SHCP. Para el caso del precio inicial del petróleo se toma el valor de la constante de un modelo autorregresivo construído con datos de: https://www.eia.gov/. Se toma el dato de la mezcla Maya.

\section{Resultados y discusión}

La combinación de ponderadores que minimiza el coeficiente de Gini de largo plazo es la siguiente: $p o n d 1 *=0.21$, pond $2 *=0.69$ y pond $3^{*}=0.10$. Esto implica, que la fórmula fiscal debería cambiar en su estructura original de la ecuación 1 (presentada en el apartado 2.1) a la ecuación 2 que se presenta a continuación:

$$
P_{i, t}=P_{i, 07}+\Delta F G P_{07, t}\left(0.21 C 1_{i, t}+0.69 C 2_{i, t}+0.100 C 3_{i, t}\right)
$$

El hallazgo de una combinación óptima, implica que si se quisiera utilizar a la FF como un mecanismo para disminuir la desigualdad del ingreso en México, se tendría que reducir la ponderación actual del criterio de desigualdad (pondl) en casi una tercera parte ( de 0.6 a 
0.21). También se tendría que aumentar la ponderación que tiene la recaudación de impuestos estatales (pond2) en más del doble (de 0.3 a 0.69), y mantener el criterio de con la misma ponderación $($ pond $3=$ pond $3 *=0.10)$.

En la tabla 2 presentamos una comparación de las variables de desempeño en cada escenario. En el escenario con combinación óptima (i.e. con los parámetros de la ecuación 2), se tiene un Gini en promedio de 0.50 , mientras que con la combinación actual de ponderadores, el Gini es de 0.52 .

Tabla 2

Variables de desempeño, escenario óptimo y escenario actual

\begin{tabular}{lcccccc}
\hline & Media & $\begin{array}{c}\text { Desviación } \\
\text { Estándar }\end{array}$ & $\begin{array}{c}\text { Coeficiente de } \\
\text { Variación }\end{array}$ & Mínimo & Máximo & Observaciones \\
\hline Combinación Óptima & 0.502 & 0.232 & 0.463 & 0.316 & 0.988 & 14500 \\
Combinación actual & 0.515 & 0.236 & 0.457 & 0.325 & 0.988 & 14500 \\
Total & 0.508 & 0.234 & 0.460 & 0.316 & 0.988 & 29000 \\
\hline
\end{tabular}

Fuente: elaboración propia con datos de la simulación

Nota: La combinación óptima es pond $1 *=0.212$, pond $2 *=0.688$, pond $3 *=0.100$, mientras que la combinación actual es pond $1 *=0.600$, pond $2 *=0.300$ y pond $3 *=0.100$.

Esto implica que un cambio en la FF, puede reducir marginalmente la desigualdad del ingreso en dos puntos porcentuales..$^{13}$ Una prueba de medias demuestra precisamente la existencia de esta diferencia (ver estadístico $t$ en la tabla 3). La desviación estándar en los dos casos es alta (poco más de 0.200 , considerando medias de alrededor de 0.500 ) y se podría argumentar que los coeficientes promedio del Gini en los dos escenarios, no deberían ser estadísticamente distintos.

\footnotetext{
${ }^{13}$ Una prueba de diferencia de medias estándar rechaza Ho a niveles de confianza altos.
} 
Tabla 3

Prueba de diferencia entre medias (asumiendo varianzas iguales)

\begin{tabular}{ccccccc}
\hline Muestra & Observaciones & Media & $\begin{array}{c}\text { Error } \\
\text { estándar }\end{array}$ & $\begin{array}{c}\text { Desviación } \\
\text { Estándar }\end{array}$ & \multicolumn{2}{c}{ Intervalo (95\% de confianza) } \\
\hline Grupo 1 & 14500 & 0.502 & 0.002 & 0.232 & 0.498 & 0.505 \\
Grupo 2 & 14500 & 0.515 & 0.002 & 0.236 & 0.511 & 0.512 \\
Combinado & 29000 & 0.510 & 0.001 & 0.234 & 0.501 & 0.511 \\
Diferencia & & -0.013 & 0.003 & & -0.019 & -0.008 \\
& & & & & & \\
& & & & & & \\
& & & & & & \\
& & & & & & \\
& & & & & & \\
\end{tabular}

Fuente: elaboración propia con datos de la simulación.

Nota: Diferencia=Media(Grupo 1)-Media(Grupo 2). Grupo 1=Grupo con la combinación óptima de ponderadores en la FF, Grupo 2=Grupo con la combinación actual.

Para tener la seguridad de que una combinación diferente de los ponderadores de la FF, genera un Gini diferente, se realiza una prueba Kolmogorov-Smirnov (KS). Dada la hipótesis nula en relación a que las distribuciones son iguales el valor del estadístico D asociado a la prueba se concluye que las distribuciones son diferentes (tabla 4).

Tabla 4

Prueba Kolmogorov-Smirnov del valor del Gini para la combinación óptima y la combinación actual

\begin{tabular}{cccc}
\hline Grupo & $\mathrm{D}$ & P-value & Corregido \\
1 & 0.065 & 0.000 & \\
2 & -0.022 & 0.001 & \\
Combinado & 0.065 & 0.000 & 0.000 \\
\hline
\end{tabular}

Fuente: elaboración propia con datos de la simulación.

Nota: se encontraron valores iguales en ambos grupos. Para el estadístico combinado hay 27959 valores únicos de un total de 29000 observaciones. 


\section{Dinámica del coeficiente de Gini}

En la figura 3, se presentan las distribuciones de frecuencia de la variable Gini para la combinación de ponderadores actual y la combinación de ponderadores optimizada. Se observa que en ambos escenarios aparecen distribuciones bimodales, lo que sugiere que es posible alcanzar baja desigualdad (i.e., entre 0.30 y 0.45 ), pero también una desigualdad alta (i.e., entre 0.90 y 1.00 ). Sin embargo, son más probables los escenarios con desigualdad de 0.30 a 0.45 en el modelo asumiendo con la combinación óptima de parámetros (la gráfica para el caso de los ponderadores optimizados es más alta).

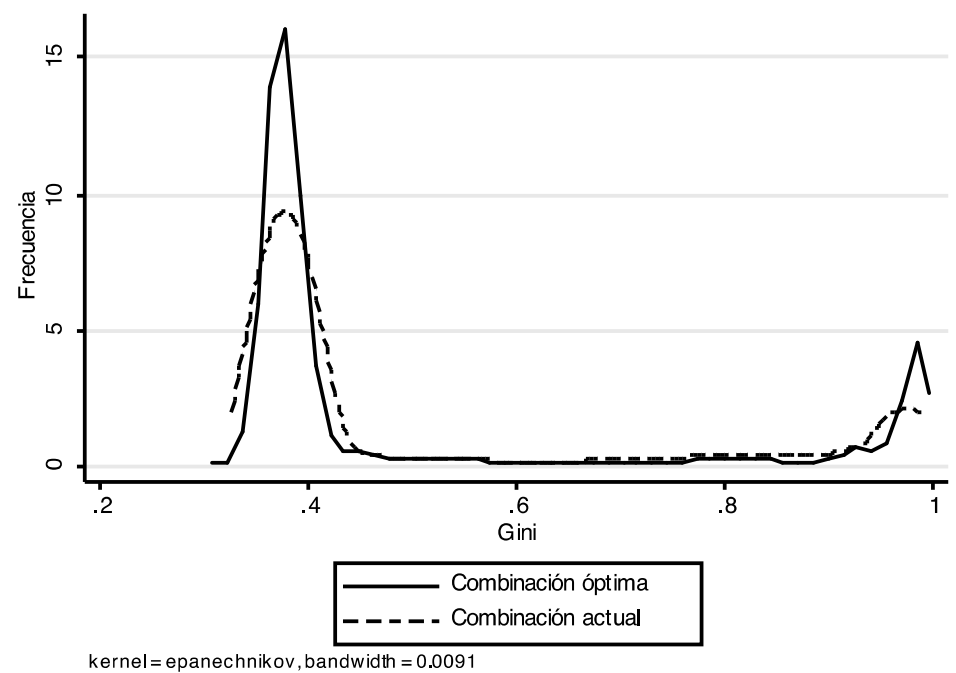

Figura 3. Distribución de frecuencia de la variable Gini.

Fuente: elaboración propia con datos de la simulación

La situación en la cual es posible alcanzar una menor desigualdad, es resultado de un comportamiento que merece un estudio a profundidad. Intuitivamente, se puede asociar a la capacidad de movilidad interestatal de los agentes (i.e. el cambio de residencia de los ciudadanos). ${ }^{14}$

\footnotetext{
${ }^{14}$ En particular deberá investigarse a qué es lo que genera el comportamiento bimodal de la desigualdad. Intuitivamente, se puede asociar este incremento al tema de la movilidad: cuando existe una mayor movilidad se deberían percibir incrementos en la desigualdad.
} 
En ambos casos, también se observa un área relativamente amplia, con baja o nula probabilidad de ocurrencia en el intervalo de valores $(0.45,0.90)$ del Gini. Esto también resulta interesante, porque implica que en el tiempo, el comportamiento del esquema de asignación actual solo puede tener dos resultados: el de una desigualdad cercana a 0.45 parecido al actual o el de una desigualdad muy alta mayor a 0.9 o cercana a 1.00 .

Adicionalmente se ha calculado el valor promedio de la variable Gini en cada uno de los períodos de las 100 simulaciones, tanto para la combinación óptima como para la combinación actual. De esta forma, ahora tenemos la trayectoria promedio que sigue la variable Gini en el tiempo para los dos escenarios. Los resultados se presentan en la Figura 4.

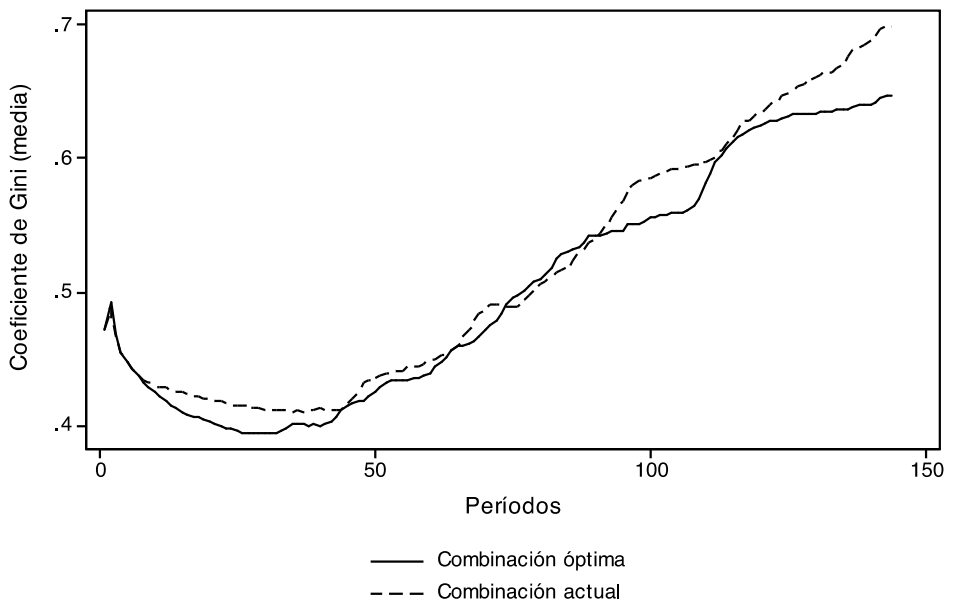

Figura 4. Comparación de la trayectoria promedio de la variable Gini para la combinación óptima y la combinación actual.

Fuente: elaboración propia con datos de la simulación

Para los primeros periodos (poco más de tres años) se observa una reducción en desigualdad en ambas combinaciones (actual y óptima) lo que implica que en sus primeros momentos el ajuste de parámetros conlleva a una menor desigualdad.

La velocidad de decaimiento es mayor en la combinación óptima al alcanzar el valor mínimo más rápido (alrededor del periodo $t=30$ ), precisamente cuando la brecha entre las dos combinaciones es más amplia. Adicionalmente hemos realizado una prueba Kolmogorov-Smirnov para la distribución del Gini en cada combinación de coeficientes de la FF. Se 
observa que ambas distribuciones son estadísticamente diferentes (ver tabla 5), aunque en la figura 4 se observa un trazo similar en particular para períodos de tiempo menores a 100.

Esto implica dos cuestiones: 1 ) en el corto plazo (i.e. antes del período $t=50$ ) ambas combinaciones generan un efecto similar en la desigualdad; 2) en el largo plazo (i.e. después del período $t=50$ ) la combinación actual en los ponderadores de la FF, tenderá a genera una mayor desigualdad que la combinación de parámetros en la FF que hemos identificado como óptima.

Tabla 5

Prueba Kolmogorov-Smirnov del valor del Gini para el promedio de la combinación óptima y la combinación actual en el tiempo

\begin{tabular}{cccc}
\hline Grupo & $\mathrm{D}$ & P-value & Corregido \\
\hline Grupo 1 & 0.193 & 0.004 & \\
Grupo 2 & -0.028 & 0.896 & \\
Combinado & 0.1931 & 0.009 & 0.001 \\
\hline
\end{tabular}

Fuente: elaboración propia con datos de la simulación

Después de que el promedio de cada combinación alcanza su valor mínimo, se observa un crecimiento pronunciado de la desigualdad del ingreso en los siguientes periodos (aproximadamente después del período $t=50$ ). La combinación óptima de ponderadores, siempre arroja menor desigualdad excepto para unos cuantos periodos alrededor de $t=80$.

Evidencia de que es posible la reducción del coeficiente de Gini en un período corto, se encuentra en el trabajo de Cortés (2013) y Cortés, \& Vargas (2017). En particular se nota que de 1989 a 2010 (i.e. 21 años) existió una reducción paulatina de la desigualdad tal como se indica en el modelo computacional.

Esta tendencia a un incremento del coeficiente de Gini en el largo plazo permite advertir tres situaciones preocupantes: 1) la fórmula de asignación de participaciones con los coeficientes actuales tenderá a generar una mayor desigualdad en el largo plazo y; 2) al utilizar los coeficientes óptimos de los ponderadores de la fórmula, se logran reducciones en la desigualdad que desafortunadamente no son sostenibles en el tiempo; 3) de mantener la combinación actual de ponderadores de la fórmula fiscal, solo se obtienen reducciones pequeñas en la desigualdad, que tampoco son sostenibles en el largo plazo. Lo anterior permite concluir que en México, la desigualdad de 
ingresos aumentará en el largo plazo, independientemente del diseño institucional que implica la fórmula fiscal. Por lo tanto, cualquier rediseño (i.e. cambios en los ponderadores) no será eficaz

\section{Conclusiones}

Los cambios institucionales en materia de federalismo fiscal de los últimos veinticinco años, han buscado una asignación más eficaz e igualitaria de los recursos para los estados del país. De acuerdo con los resultados del modelo computacional, es posible reflejar el comportamiento de la desigualdad en México. En torno a la hipótesis de trabajo: "la combinación actual de ponderadores de la Fórmula de Coordinación Fiscal no contribuye a la reducción en el nivel de desigualdad de los mexicanos", se concluye que con el modelo computacional se tienen los argumentos empíricos para no rechazarla.

Al mismo tiempo, se demuestra que de mantener esta combinación en los ponderadores, la desigualdad en México en el largo plazo no se reduce y por el contrario aumentará considerablemente. En las simulaciones se observó un efecto de "J" (ver figura 4), donde en un lapso de menos de 50 períodos, se podría observar una paulatina reducción de la desigualdad. Esta reducción en el mejor de los casos podría ubicar a México con un Gini un poco menor al 0.40. La reducción sería mayor, si se decidiera utilizar la combinación óptima de parámetros que se encuentra con los algoritmos de optimización del modelo.

Sin embargo, pasando el lapso de reducción de la desigualdad de la Figura 4 (i.e. 50 períodos), se esperaría un aumento considerable de la desigualdad, independientemente de si se mantiene la combinación actual de coeficientes, o de si se utiliza la combinación óptima de coeficientes que estima el modelo. Este resultado además de desalentador, permite sostener que se debe plantear otro diseño institucional que trascienda la definición de ponderadores. Los aspectos normativos actuales en la Ley de Coordinación Fiscal, no favorecen la reducción de desigualdad.

Parte de la explicación de este fenómeno, se puede relacionar con que los cambios en la distribución de recursos, tienen efectos sobre el crecimiento de las entidades federativas. Todo cambio en la cantidad de ingresos de un estado, modifica el gasto público estatal y el PIB. Por el diseño de la Fórmula Fiscal que integra el PIB como una variable para asignar recursos, si se incide positiva o negativamente en el PIB a través del gasto público, existirán repercusiones nuevamente en el reparto de participaciones. De esta forma, se genera un proceso de retroalimentación positiva.

Usando un modelo computacional, se aporta evidencia que soporta algunos argumentos del debate en materia de descentralización fiscal y desigualdad. En concreto, se demuestra que un cambio que privilegie el criterio de eficiencia recaudatoria, i.e., dé impulso a la des- 
centralización de recursos, mejorará el bienestar de los ciudadanos a nivel individual. Esta mejora también repercutirá en una reducción a nivel social (al menos de corto plazo) de la desigualdad. Lo anterior es consistente con el argumento de Tselios et al (2011) y Sepúlveda y Martínez-Vázquez (2011), donde se encontró que la descentralización fiscal reducía la desigualdad.

Sin embargo, como ya se ha comentado, dado el efecto de "J" de la figura 4, no se puede indicar que la descentralización fiscal que implica el modelo Tiebout, genere una reducción en desigualdad de forma sostenible a largo plazo. Por lo tanto, se prueba también que los procesos de descentralización tienen efectos negativos en la distribución del ingreso, generando mayor desigualdad tal cual lo plantea Tanzi (1996) y Zhang (2006).

Dentro de los subproductos que podría obtenerse a partir del modelo, es la posibilidad de analizar escenarios concretos relacionados con el impacto de movimientos en distintas variables. Por ejemplo, el impacto de la desigualdad ante cambios en precios del petróleo (i.e. tendencias al alza o a la baja), ante la definición de nuevos impuestos o eliminación de los ya existentes, o bien, ante el incremento en la movilidad de las personas por situaciones exógenas al modelo (e.g. ante el incremento de la violencia). Adicionalmente, sería de interés particular, explorar el comportamiento del crecimiento económico de las entidades federativas ante cambios en la ponderación de la Fórmula Fiscal.

Una de las principales limitaciones del trabajo es que no incluye un análisis sobre el financiamiento que obtienen los estados del país a partir de los Fondos de Aportaciones. Es deseable incorporar también a futuro el efecto de este tipo de mecanismos de asignación de recursos.

\section{Referencias}

Bardhan, P. (2002). "Decentralization of governance and development". Journal of Economic Perspective, 16(4), 185-205. https://doi.org/10.1257/089533002320951037.

Blöchliger, H. (2014). "Fiscal Equalisation -A Cross-Country Perspective." OECD Network on Fiscal Relations across Government Levels. París: OCDE. https://doi.org.10.3326/pse.41.2.6.

Carmona Guerrero, N. \& Caamal-Olvera, C. G. (2018). “¿Las transferencias federales han logrado reducir la desigualdad en las entidades federativas en México?”. EconoQuantum, 15(1),31-51.http://dx.doi.org/10.18381/ eq.v15i1.7111.

Careaga, Maite \& Weingast, B. R. (2003). "Fiscal Federalism, Good Governance, and Economic Growth in Mexico", 399-435, en Rodrick, D. (2003), en In search of prosperity: analytical narratives on economic growth. New Jersey: Princeton University Press. https://doi.org/10.1515/9781400845897.

Cortés, F. (2013). "Medio siglo de desigualdad en el ingreso en México". Economía UNAM, 10(29), 12-34. https:// doi.org/10.1016/S1665-952X(13)72193-5 
Cortés, F., \& Vargas, D. (2017). La evolución de la desigualdad en México: viejos y nuevos resultados. Revista de Economía Mexicana, 2, 39-96.

Chavez-Presa, J.A. (2004). "Los Límites del Federalismo. El reto del Desarrollo Local”. Estudios sobre Desarrollo Humano, PNUD México, No. 2004-9. Disponible en: http://www.undp.org.mx/IMG/pdf/ChavezPresa2004. pdf. https://doi.org/10.18356/b1343da7-es. Consultado: 15/05/2020.

Díaz-Cayeros, A. (2004). "Dependencia fiscal y estrategias de coalición en el federalismo mexicano". Política y Gobierno, 11(2), 229-262.

Dowding, K., P. John \& S. Biggs (1994). “Tiebout: A Survey of the Empirical Literature”. Urban Studies, 31, 767797. https://doi.org/10.1080/00420989420080671.

Eifert, B., Gelb, A. \& Tallroth, N.B. (2003). "The political economy of fiscal policy and economic management in oil-exporting countries”, en Davis, J.M., Ossowski, R. \& Fedelino, A. (2003). Fiscal policy formulation and implementation in oil-producing countries, 82-122, Washington, D.C.: Fondo Monetario Internacional. https:// doi.org/10.1596/1813-9450-2899.

Figueroa, F. C. (2014). "El Sistema Nacional de Coordinación Fiscal (SNFC) y sus efectos en el endeudamiento y el esfuerzo fiscal del Distrito Federal y de las entidades del país, 1995-2010”. Revista Análisis Económico, 29(71), 79-111. https://doi.org/10.29201/pe-ipn.v12i23.103.

Hernández-Trillo, F. (2011). "Manejo de Riesgos Financieros en Entidades Federativas en México". En Mendoza-Velázquez, A. (2011). Las Finanzas Públicas Estatales en México: Herramientas de Diagnóstico y Respuesta en un Entorno de Crísis. Mexico, D.F : Porrúa.

Hoyt, W. H. (1991). "Property taxation, Nash equilibrium, and market power". Journal of Urban Economics, 30(1), Julio 1991, 123-131. https://doi.org/10.1016/0094-1190(91)90049-D.

Ibarra-Salazar, J. (2018). Fundamentos de la nueva fórmula de asignación del Fondo de Aportaciones para la Infraestructura Social en México. Trimestre Económico, 85(337), 195-218. http://dx.doi.org/10.20430/ete. v85i337.664

Kollman, K., J.H. Miller \& S.E. Page (1997). "Political institutions and sorting in a Tiebout model". The American Economic Review, 87(5), 977-992. Disponible en: https://www.jstor.org/stable/2951336. Consultado: $15 / 05 / 2020$.

Lessmann, C. (2009). "Fiscal decentralization and regional disparity: Evidence from cross-section and panel data". Dresden discussion paper series in economics, 8(9). http://dx.doi.org/10.2139/ssrn.936874

Lessmann, C. (2012). "Regional inequality and decentralization: an empirical analysis," Environment and Planning, 44(6), 1363-1388, https://doi.org/10.1068/a44267.

Musgrave, R. A. y P. B. Musgrave (1989). "Public finance in theory and practice". Quinta edición, New York: Mcgraw Hill.

Nishida, R., T. Yamada , A. Yoshikawa y T. Terano (2011). Identification of Voting with Individual's Feet Through Agent-Based Modeling. Agent-Based Approaches in Economic and Social Complex Systems VI, 119-132. https://doi.org/10.1007/978-4-431-53907-0_9.

Oates, W. E. (1968). "The Theory of Public Finance in a Federal System”. The Canadian Journal of Economics / Revue canadienne d'Economique, (1)( 1), 37-54. https://doi.org/10.2307/133460.

Oates, W. (2005), "Towards a Second-generation Theory of Fiscal Federalism", International Tax and Public Finance, 12(4), 349-373. https://doi.org/10.1007/s10797-005-1619-9.

OCDE (2007). "Economic survey of Mexico 2007: Putting public finances on a firmer footing.". Policy Brief, Octubre 2007. https://doi.org/10.1787/eco_surveys-mex-2007-4-en.

Orfield, M. (2002). "Metropolitics: Coalitions for Regional Reform". The Brookings Review, (15)(1), 6-9. https:// doi.org/10.2307/20080701.

Page S.E. (2010). Diversity and Complexity (Primers in Complex Systems). Princeton University Press; Primera edición (Noviembre 8, 2010).

Penn, E.M. (2004).“Institutions and sorting in a model of metropolitan fragmentation”. Complexity, 9(5), 62-70. https://doi.org/10.1002/cplx.20039. 
Prud'homme, R. (1995). "The dangers of decentralization". World Bank Research Observer, 10, 201-220. https:// doi.org/10.1093/wbro/10.2.201.

Rodríguez-Pose, A. y Krøijer, A. (2009). "Fiscal decentralization and economic growth in Central and Eastern Europe". Growth and change, 40(3), 387-417. https://doi.org/10.1111/j.1468-2257.2009.00488.x.

Rodríguez-Pose, A. y R. Ezcurra (2010). "Is fiscal decentralization harmful for economic growth? Evidence from the OECD countries”. Journal of Economic Geography, 11(4), 619-643. https://doi.org/10.1093/jeg/lbq025.

Rodríguez-Pueblita, J. C. (2017). Transferencias intergubernamentales y disparidades fiscales entre los estados en México. Documento para discusión No. IDB-DP-495, Banco Interamericado de Desarrollo . http://dx.doi. org/10.18235/0000874\#sthash.YVJfh4hg.dpuf

Rodríguez, R. H. (2006). La disputa por el presupuesto federal. Presidencialismo y gobiernos estatales en México. Foro internacional, 46(1), 103-121. Disponible en: http://forointernacional.colmex.mx/index.php/fi/article/ download/1810/1800. Consultado: 15/05/2020

Sakashita, N. (1999). "Dynamic Analysis of the Tiebout Model with Numerical Simulations". The Japanese Economic Review, 50(11), 62-75. https://doi.org/10.1111/1468-5876.00103.

Ibarra- Salazar, J., Musi, A. S., \& Cervantes, L. S. (1999). México: ingresos estatales y

dependencia de las participaciones federales. Comercio exterior, 438-444. Disponible en: http://revistas.bancomext.gob.mx/rce/magazines/282/3/RCE3.pdf. Consultado: 15/05/2020

Schwartz, E.S. (1997). "The stochastic behavior of commodity prices: Implications for valuation and hedging", The Journal of Finance, 52(3), 923-973. https://doi.org/10.1111/j.1540-6261.1997.tb02721.x.

Sepúlveda, C. \& J. Martinez-Vazquez (2011). "Explaining Property Tax Collections in Developing Countries: The Case of Latin America", en Brosio, G. \& Jiménez, J.P. Decentralization and Reform in Latin America. Massachusetts: Edward Elgar Publishing, Inc. https://doi.org/10.4337/9781781006269.00013.

Sobarzo Fimbres, H. (2004). "Federalismo fiscal en México". Economía, sociedad y territorio, Dossier especial, 103-121. Recuperado de: https://www.redalyc.org/articulo.oa?id=111/11109905

Tanzi, V. (1996). "Fiscal federalism and decentralization: a review of some eficiency and macroeconomic aspects", en Bruno M. \& Pleskovic, B. Annual World Bank conference on economic development, 539-567.Washington D.C.: World Bank. https://doi.org/10.1596/0-8213-3786-6.

Tiebout, C.M. (1956). "A Pure Theory of Local Expenditures". The Journal of Political Economy, 64, (5), 416-424. https://doi.org/10.1086/257839.

Tselios, V., Rodríguez-Pose, A., Pike, A., Tomaney, J., \& Torrisi, G. (2012). "Income inequality, decentralisation, and regional development in Western Europe”. Environment and Planning A, 44(6), 1278-1301. https://doi. org/10.1068/a44334.

Von Neumann, J. \& Burks, A. W. (1966). Theory of self-reproducing automata. Urbana: University of Illinois Press. Disponible en: http://www.cba.mit.edu/events/03.11.ASE/docs/VonNeumann.pdf. Consultado: 15/05/2020.

Weingast, B. R. (2009). Second generation fiscal federalism: The implications of fiscal incentives. Journal of Urban Economics, 65(3), 279-293. https://doi.org/10.1016/j.jue.2008.12.005.

West, L. A. \& C. Wong (1995). "Fiscal decentralization and growing regional disparities in rural China: some evidence in the provision of social services". Oxford Review of Economic Policy 11(4), 70-84. https://doi. org/10.1093/oxrep/11.4.70.

Zhang, X. (2006). "Fiscal decentralization and political centralization in China : implications for growth and inequality". World Institute for Development Economics Research. United Nations University. Documento de Investigación no. 2006/93. https://doi.org/10.1016/j.jce.2006.08.006 